\title{
A novel kind of Hermite based Frobenius type Eulerian polynomials
}

\author{
Waseem Ahmad Khan ${ }^{1}$, Kottakkaran Sooppy Nisar ${ }^{2}$, Mehmet Acikgoz ${ }^{3}$ and Ugur Duran ${ }^{4, *}$ \\ ${ }^{1}$ Department of Mathematics, Faculty of Science, \\ Integral University, Lucknow-226026, India, \\ E-Mail: waseem08_khan@rediffmail.com
}

\author{
${ }^{2}$ Department of Mathematics, College of Arts and Science-Wadi Al dawaser, \\ Prince Sattam bin Abdulaziz University, Riyadh region 11991, Saudi Arabia \\ E-Mail: n.sooppy@psau.edu.sa \\ ${ }^{3}$ University of Gaziantep, Faculty of Science and Arts, \\ Department of Mathematics, TR-27310 Gaziantep, Turkey \\ E-Mail: acikgoz@gantep.edu.tr \\ ${ }^{4}$ Department of the Basic Concepts of Engineering, \\ Faculty of Engineering and Natural Sciences, \\ Iskenderun Technical University, TR-31200 Hatay, Turkey \\ E-Mail: mtdrnugur@gmail.com \& ugur.duran@iste.edu.tr \\ *Corresponding Author
}

\begin{abstract}
We introduce a new kind of extended Hermite-based Frobenius type Eulerian polynomials and then derive diverse explicit and implicit summation equations including some symmetric formulas by utilizing series manupulation method. Multifarious summation formulas and identities given earlier for some well known polynomials such as Eulerian polynomials and Frobenius type Eulerian polynomials are generalized.
\end{abstract}

2010 Mathematics Subject Classification. Primary 11B68, Secondary 33C45, 11Y16.

Key Words and Phrases. Hermite polynomials, Frobenius type Eulerian polynomials, Hermite-based Frobenius type Eulerian polynomials, Summation formulae, Symmetric identities.

\section{INTRODUCTION}

Special polynomials including the reputed Bernoulli, Hermite and Euler polynomials and also their generalizations with many applications have been investigated extensively and studied by many mathematicians and also physicists, $c f$. [1-19]. For instance, Carlitz [2,3] introduced and investigated the Eulerian polynomials and higher order Eulerian polynomials, which are closely related with the Euler and Bernoulli numbers and polynomials. Choi et al. [4] gave some formulae for the Frobenius-Euler numbers and polynomials via the fermionic $p$-adic integral methods. Dattoli et al. [5] defined new forms of Bernoulli and Euler numbers and polynomials by applying the series manipulation method, which are exploited to get further classes of partial sums including generalized many index many variable polynomials. Khan [6] researched and acquired some behaviors of the extended Apostol type Hermite-based polynomials. Kim et al. [7, 8] derived several relations for the Eulerian polynomials in connection with Bernoulli, Euler, tangent and Genocchi numbers. Kim et al. [9] provided a lot of new identities for the Frobenius-Euler polynomials and numbers. Kurt et al. $[10,11]$ derived several new formulas for the Frobenius-Euler numbers and polynomials including generalized Hurwitz-Lerch zeta function at negative integers, some of which are generalizations of known Carlitz's results. Pathan et al. [12-15] studied and investigated multifarious symmetric identities and summation formulas for the some extended Hermite based polynomials covering Hermite-based Apostol Bernoulli polynomials, 
Hermite-based Apostol Euler polynomials, Hermite-based Apostol Genocchi polynomials. Srivastava [16] investigated various general families of hypergeometric polynomials and their associated single-, double-, and triple-integral representations of the Eulerian and other types, which are related to the Laguerre, Jacobi, Bessel and Hermite polynomials. Srivastava et al. proved some explicit identities associated with the Frobenius-type Eulerian polynomials in view of the generalized Stirling numbers of the second kind and derive an explicit formula for the tangent numbers of higher order. Simsek [19] constructed novel exponential generating functions for extended Eulerian type polynomials and numbers in order to provide a novel approach to derive relationships involving recurrence relations and multiplication identities for these numbers and polynomials by using the differential and functional equations.

The Hermite Kampé de Fériet polynomials (2VHKdFP) $H_{n}(x, y)$ with two parameters, cf. [1, 5], are defined as

$$
H_{n}(x, y)=n ! \sum_{r=0}^{\left[\frac{n}{2}\right]} \frac{y^{r} x^{n-2 r}}{r !(n-2 r) !} .
$$

It is readily seen by (1.1) that

$$
H_{n}(2 x,-1)=H_{n}(x) \text { and } H_{n}\left(x,-\frac{1}{2}\right)=H e_{n}(x),
$$

where $H_{n}(x)$ and $H e_{n}(x)$ are known as ordinary Hermite polynomials. Also, we have

$$
H_{n}(x, 0)=x^{n} .
$$

The exponential generating function of the Hermite Kampé de Fériet polynomials of two parameters is given by (see $[6,12-15])$ :

$$
e^{x t+y t^{2}}=\sum_{n=0}^{\infty} H_{n}(x, y) \frac{t^{n}}{n !} .
$$

The Frobenius type Eulerian polynomials $A_{n}^{(\alpha)}(x ; \lambda)$ of order $\alpha \in \mathbb{C}$ are defined by means of the following exponential generating function as follows (see $[2,3,7,8,9,18]$ ):

$$
\left(\frac{1-\lambda}{e^{t(\lambda-1)}-\lambda}\right)^{\alpha} e^{x t}=\sum_{n=0}^{\infty} A_{n}^{(\alpha)}(x ; \lambda) \frac{t^{n}}{n !},
$$

where $\lambda$ is a complex number with $\lambda \neq 1$. The corresponding numbers $A_{n}^{(\alpha)}$ of the Frobenius-type Eulerian polynomials are computed by

$$
A_{n}^{(\alpha)}(\lambda)=A_{n}^{(\alpha)}(0 ; \lambda)
$$

which are called the Frobenius type Eulerian numbers (see $[2,3])$. Clearly, we have

$$
A_{n}^{(\alpha)}(x ; \lambda)=\sum_{m=0}^{n}\left(\begin{array}{c}
n \\
m
\end{array}\right) A_{m}^{(\alpha)}(\lambda) x^{n-m} .
$$

The classical Eulerian polynomials $A_{n}(\lambda)$ given by

$$
A_{n}(\lambda)=A_{n}^{(1)}(0 ; \lambda)
$$

are defined by the following generating function:

$$
\frac{1-\lambda}{e^{t(\lambda-1)}-\lambda}=\sum_{n=0}^{\infty} A_{n}(\lambda) \frac{t^{n}}{n !}
$$

and can be computed inductively as follows:

$$
A_{0}(\lambda)=1 \text { and } A_{n}(\lambda)=\sum_{m=0}^{n-1}\left(\begin{array}{c}
n \\
m
\end{array}\right) A_{m}(\lambda)(\lambda-1)^{n-m-1}, n \geq 1 .
$$

These numbers play a significant role in combinatorial and number theory. Many authors investigated the Frobenius type Eulerian polynomials (see $[4,10,11,16,19]$ ). An application to the normal ordering of expressions involving bosonic annihilation and creation operators in [9]. 
The Apostol type Bernoulli polynomials $B_{n}^{(\alpha)}(x ; \lambda)$ of order $\alpha$, the Apostol type Euler polynomials $E_{n}^{(\alpha)}(x ; \lambda)$ of order $\alpha$ and the Apostol type Genocchi polynomials $G_{n}^{(\alpha)}(x ; \lambda)$ of order $\alpha$ are defined by means of the following generating function (see [6, 12-15]):

$$
\begin{aligned}
& \left(\frac{t}{\lambda e^{t}-1}\right)^{\alpha} e^{x t}=\sum_{n=0}^{\infty} B_{n}^{(\alpha)}(x ; \lambda) \frac{t^{n}}{n !},|t+\log \lambda|<2 \pi, 1^{\alpha}=1, \\
& \left(\frac{2}{\lambda e^{t}+1}\right)^{\alpha} e^{x t}=\sum_{n=0}^{\infty} E_{n}^{(\alpha)}(x ; \lambda) \frac{t^{n}}{n !}, \quad|t+\log \lambda|<\pi, 1^{\alpha}=1 \\
& \left(\frac{2 t}{\lambda e^{t}+1}\right)^{\alpha} e^{x t}=\sum_{n=0}^{\infty} G_{n}^{(\alpha)}(x ; \lambda) \frac{t^{n}}{n !}, \quad|t+\log \lambda|<\pi, 1^{\alpha}=1 .
\end{aligned}
$$

Clearly, we have

$$
B_{n}^{(\alpha)}(\lambda)=B_{n}^{(\alpha)}(0 ; \lambda), E_{n}^{(\alpha)}(\lambda)=E_{n}^{(\alpha)}(0 ; \lambda), G_{n}^{(\alpha)}(\lambda)=G_{n}^{(\alpha)}(0 ; \lambda)
$$

Pathan and Khan [13] introduced the generalized Hermite-Bernoulli polynomials of two variables ${ }_{H} B_{n}^{(\alpha)}(x, y)$ defined by

$$
\left(\frac{t}{e^{t}-1}\right)^{\alpha} e^{x t+y t^{2}}=\sum_{n=0}^{\infty}{ }_{H} B_{n}^{(\alpha)}(x, y) \frac{t^{n}}{n !},
$$

which is clearly a extension of Bernoulli polynomials, Bernoulli numbers, usual Hermite polynomials. For $\alpha=1$, we get the Hermite-Bernoulli polynomials ${ }_{H} B_{n}(x, y)$ considered by Dattoli et al [5, p.386(1.6)] in the form

$$
\left(\frac{t}{e^{t}-1}\right) e^{x t+y t^{2}}=\sum_{n=0}^{\infty}{ }_{H} B_{n}(x, y) \frac{t^{n}}{n !} .
$$

The Stirling numbers of the second kind is defined via the following generating function (see $[16,19])$ :

$$
\left(e^{t}-1\right)^{n}=n ! \sum_{l=n}^{\infty} S_{2}(l, n) \frac{t^{l}}{l !},
$$

and its first kind is given by

$$
(x)_{n}=x(x-1) \cdots(x-n+1)=\sum_{l=0}^{n} S_{1}(l, n) x^{l} .
$$

The reminder of the paper is organized as follows. We give a brief review of Hermite-based Frobenius type Eulerian polynomials ${ }_{H} A_{n}^{(\alpha)}(x, y ; \lambda)$ and their properties. Several general symmetry identities and implicit summation formulae are acquired by utilizing different analytical means and applying series manipılation method.

\section{Hermite-based Frobenius type Eulerian polynomials ${ }_{H} A_{n}^{(\alpha)}(x, y ; \lambda)$}

In this section, we firstly introduce Hermite-based Frobenius type Eulerian polynomials $(\mathrm{HbFtEp})_{H} A_{n}^{(\alpha)}(x, y ; \lambda)$ and explicit formula for the Frobenius type Eulerian polynomials and investigate its properties. Now we start at the following definition.

Definition 2.1. For $\lambda \in \mathbb{C}$, the generalized Hermite-based Frobenius type Eulerian polynomials ${ }_{H} A_{n}^{(\alpha)}(x, y ; \lambda)$ of order $\alpha$ are defined by means of the following generating function:

$$
\left(\frac{1-\lambda}{e^{t(\lambda-1)}-\lambda}\right)^{\alpha} e^{x t+y t^{2}}=\sum_{n=0}^{\infty}{ }_{H} A_{n}^{(\alpha)}(x, y ; \lambda) \frac{t^{n}}{n !} .
$$


When $x=y=0$ in $(2.1),{ }_{H} A_{n}^{(\alpha)}(0,0 ; \lambda)=A_{n}^{(\alpha)}(\lambda)$ are called the $n^{t h}$ Frobenius type Eulerian numbers of order $\alpha$.

For $x=0$ in $(2.1)$, we get

$$
{ }_{H} A_{n}^{(\alpha)}(0, y ; \lambda)=\sum_{m=0}^{\left[\frac{n}{2}\right]} A_{n-2 m}^{(\alpha)}(\lambda) y^{m} \frac{n !}{(n-2 m) !} .
$$

Theorem 2.1. The following series representation for the Hermite-based Frobenius type Eulerian polynomials ${ }_{H} A_{n}^{(\alpha)}(x, y ; ; \lambda)$ of order $\alpha$ holds true:

$$
(\lambda-1)^{-n}{ }_{H} A_{n}^{(\alpha)}\left((\lambda-1) x,(\lambda-1)^{2} y ; \lambda\right)={ }_{H} E_{n}^{(\alpha)}(x, y ; \lambda) .
$$

Proof. Consider the generating function (2.1), we have

$$
\sum_{n=0}^{\infty} \frac{{ }_{H} A_{n}^{(\alpha)}\left((\lambda-1) x,(\lambda-1)^{2} y ; \lambda\right)}{n !} \frac{t^{n}}{(\lambda-1)^{n}}=\left(\frac{1-\lambda}{e^{t}-\lambda}\right)^{\alpha} e^{x t+y t^{2}}=\sum_{n=0}^{\infty}{ }_{H} E_{n}^{(\alpha)}(x, y ; \lambda) \frac{t^{n}}{n !},
$$

where ${ }_{H} E_{n}^{(\alpha)}(x, y ; \lambda)$ is called the Hermite-based Frobenius-Euler polynomials, which is defined by Pathan and Khan [15] and comparing the coefficients of $t^{n}$, we arrive at the required result (2.3).

Theorem 2.2. The following series representation for the Hermite-based Frobenius type Eulerian polynomials ${ }_{H} A_{n}^{(\alpha)}(x, y ; ; \lambda)$ of order $\alpha$ holds true:

$$
{ }_{H} A_{n}^{(\alpha)}(x, y ; \lambda)=\sum_{m=0}^{n}\left(\begin{array}{c}
n \\
m
\end{array}\right) A_{n-m}^{(\alpha)}(\lambda) H_{m}(x, y) .
$$

Proof. Using equation (1.2) and (1.5) in the left hand side of equation (2.1) and then applying the Cauchy product rule and equating the coefficients of same powers of $t$ in both sides of resultant equation, we get representation (2.4).

Theorem 2.3. The following summation formulas for the Hermite-based Frobenius type Eulerian polynomials ${ }_{H} A_{n}^{(\alpha)}(x, y ; ; \lambda)$ of order $\alpha$ holds true:

$$
{ }_{H} A_{n}^{(\alpha+\beta)}(x+w, y+u ; \lambda)=\sum_{m=0}^{n}\left(\begin{array}{c}
n \\
m
\end{array}\right){ }_{H} A_{m}^{(\beta)}(u, w ; \lambda)_{H} A_{n-m}^{(\alpha)}(x, y ; \lambda)
$$

and

$$
{ }_{H} A_{n}^{(\alpha)}(x+z, y ; \lambda)=\sum_{m=0}^{n}\left(\begin{array}{c}
n \\
m
\end{array}\right) A_{n-m}^{(\alpha)}(x ; \lambda) H_{m}(y, z) .
$$

Proof. Applying Definition (2.1), we have

$$
\begin{aligned}
\sum_{n=0}^{\infty}{ }_{H} A_{n}^{(\alpha+\beta)}(x+y, z+w ; \lambda) \frac{t^{n}}{n !} & =\left(\frac{1-\lambda}{e^{t(\lambda-1)}-\lambda}\right)^{\alpha+\beta} e^{(x+w) t+(y+u) t^{2}} \\
& =\sum_{n=0}^{\infty} H_{n}^{(\alpha)}(x, y ; \lambda) \frac{t^{n}}{n !} \sum_{m=0}^{\infty} H_{H}^{(\beta)}(u, w ; \lambda) \frac{t^{m}}{m !} \\
& =\sum_{n=0}^{\infty} \sum_{m=0}^{n}\left(\begin{array}{c}
n \\
m
\end{array}\right)_{H} A_{m}^{(\beta)}(u, w ; \lambda)_{H} A_{n-m}^{(\alpha)}(x, y ; \lambda) \frac{t^{n}}{n !} .
\end{aligned}
$$

Now equating the coefficients of the like powers of $t$ in the above equation, we get the result (2.5). By utilizing the same proof method above, the Eq. (2.6) can be shown. 
Theorem 2.4. The following recursive formulas for the Hermite-based Frobenius type Eulerian polynomials of order $\alpha$ holds true:

$$
\frac{\partial_{H} A_{n}^{(\alpha)}(x, y ; \lambda)}{\partial x}=n_{H} A_{n-1}^{(\alpha)}(x, y ; \lambda) \text { and } \frac{\partial_{H} A_{n}^{(\alpha)}(x, y ; \lambda)}{\partial y}=n(n-1)_{H} A_{n-2}^{(\alpha)}(x, y ; \lambda) .
$$

Proof. The proof follows from (2.3). So we omit them.

Theorem 2.5. The following relation for the Hermite-based Frobenius type Eulerian polynomials ${ }_{H} A_{n}^{(\alpha)}(x, y ; j \lambda)$ of order $\alpha$ holds true:

$$
\begin{aligned}
& (2 \lambda-1) \sum_{k=0}^{n}\left(\begin{array}{c}
n \\
m
\end{array}\right) A_{k}(x ; \lambda)_{H} A_{n-k}\left(\frac{y \lambda}{\lambda-1}, z \frac{\lambda^{2}}{(\lambda-1)^{2}} ; 1-\lambda\right) \frac{(\lambda-1)^{n-k}}{\lambda^{n-k}} \\
= & \lambda_{H} A_{n}(x+y, z ; \lambda)-(1-\lambda)_{H} A_{n}\left(\frac{(x+y) \lambda}{\lambda-1}, z \frac{\lambda^{2}}{(\lambda-1)^{2}} ; 1-\lambda\right) \frac{(\lambda-1)^{n}}{\lambda^{n}} .
\end{aligned}
$$

Proof. We set

$$
\frac{2 \lambda-1}{\left(e^{t(\lambda-1)}-\lambda\right)\left(e^{t(\lambda-1)}-(1-\lambda)\right)}=\frac{1}{e^{t(\lambda-1)}-\lambda}-\frac{1}{e^{t(\lambda-1)}-(1-\lambda)} .
$$

From the equation $(2.9)$, we see that

$$
\begin{aligned}
(2 \lambda-1) \frac{(1-\lambda) e^{x t}(1-(1-\lambda)) e^{y t+z t^{2}}}{\left(e^{t(\lambda-1)}-\lambda\right)\left(e^{t(\lambda-1)}-(1-\lambda)\right)}= & \frac{(1-\lambda) e^{y t+z t^{2}} \lambda e^{x t}}{e^{t(\lambda-1)}-\lambda} \\
& -\frac{(1-\lambda) e^{y t+z t^{2}} \lambda e^{x t}(1-(1-\lambda))}{e^{t(\lambda-1)}-(1-\lambda)}
\end{aligned}
$$

and then

$$
\begin{gathered}
(2 \lambda-1) \frac{(1-\lambda) e^{x t}}{\left(e^{t(\lambda-1)}-\lambda\right)} \frac{(1-(1-\lambda)) e^{\frac{y \lambda}{\lambda-1} \frac{t(\lambda-1)}{\lambda}+z \frac{\lambda^{2}}{(\lambda-1)^{2}}\left(\frac{t(\lambda-1)}{\lambda}\right)^{2}}}{e^{\frac{t(\lambda-1)}{\lambda} \lambda}-(1-\lambda)}=\frac{(1-\lambda) e^{y t+z t^{2}} \lambda e^{x t}}{e^{t(\lambda-1)}-\lambda} \\
-(1-\lambda) \frac{e^{\frac{(x+y) \lambda}{\lambda-1} \frac{t(\lambda-1)}{\lambda}+z \frac{\lambda^{2}}{(\lambda-1)^{2}}\left(\frac{t(\lambda-1)}{\lambda}\right)^{2}} \lambda(1-(1-\lambda))}{e^{\frac{t(\lambda-1)}{\lambda} \lambda}-(1-\lambda)},
\end{gathered}
$$

which on using equations (1.5) and (2.1) in both sides, we have

$$
\begin{aligned}
& (2 \lambda-1)\left(\sum_{k=0}^{\infty} A_{k}(x ; \lambda) \frac{t^{k}}{k !}\right)\left(\sum_{n=0}^{\infty}{ }_{H} A_{n}\left(\frac{y \lambda}{\lambda-1}, z \frac{\lambda^{2}}{(\lambda-1)^{2}} ; 1-\lambda\right) \frac{(\lambda-1)^{n}}{\lambda^{n}} \frac{t^{n}}{n !}\right) \\
= & \lambda \sum_{n=0}^{\infty} H_{H} A_{n}(x+y, z ; \lambda) \frac{t^{n}}{n !}-(1-\lambda) \sum_{n=0}^{\infty} H_{n}\left(\frac{(x+y) \lambda}{\lambda-1}, z \frac{\lambda^{2}}{(\lambda-1)^{2}} ; 1-\lambda\right) \frac{(\lambda-1)^{n}}{\lambda^{n}} \frac{t^{n}}{n !} .
\end{aligned}
$$

Applying the Cauchy product rule in the above equation and then equating the coefficients of like powers of $t$ in both sides of the resultant equation, assertion (2.8) follows.

Theorem 2.6. The following relation for the Hermite-based Frobenius-type Eulerian polynomials ${ }_{H} A_{n}^{(\alpha)}(x, y ; \lambda)$ of order $\alpha$ holds true:

$$
\lambda_{H} A_{n}(x, y ; \lambda)=\sum_{k=0}^{n}\left(\begin{array}{c}
n \\
m
\end{array}\right){ }_{H} A_{n-k}(x, y ; \lambda)(\lambda-1)^{k}-(1-\lambda) H_{n}(x, y) .
$$

Proof. Consider the following identity

$$
\frac{\lambda}{\left(e^{t(\lambda-1)}-\lambda\right) e^{t(\lambda-1)}}=\frac{1}{\left(e^{t(\lambda-1)}-\lambda\right)}-\frac{1}{e^{t(\lambda-1)}} .
$$


Evaluating the following fraction using identity (2.11), we find

$$
\begin{gathered}
\frac{\lambda(1-\lambda) e^{x t+y t^{2}}}{\left(e^{t(\lambda-1)}-\lambda\right) e^{t(\lambda-1)}}=\frac{(1-\lambda) e^{x t+y t^{2}}}{\left(e^{t(\lambda-1)}-\lambda\right)}-\frac{(1-\lambda) e^{x t+y t^{2}}}{e^{t(\lambda-1)}} \\
\lambda \sum_{n=0}^{\infty}{ }_{H} A_{n}(x, y ; \lambda) \frac{t^{n}}{n !}=\sum_{n=0}^{\infty} H_{H} A_{n}(x, y ; \lambda) \frac{t^{n}}{n !} \sum_{k=0}^{\infty}(\lambda-1)^{k} \frac{t^{k}}{k !}-(1-\lambda) \sum_{n=0}^{\infty} H_{n}(x, y) \frac{t^{n}}{n !} .
\end{gathered}
$$

Applying the Cauchy product rule in the above equation and then equating the coefficients of like powers of $t$ in both sides of the resultant equation, assertion (2.10) follows.

Theorem 2.7. The following relation for the Hermite-based Frobenius-type Eulerian polynomials ${ }_{H} A_{n}^{(\alpha)}(x, y ; \lambda)$ of order $\alpha$ holds true:

$$
{ }_{H} A_{n}^{(\alpha)}(x, y ; \lambda)=\frac{1}{1-\lambda} \sum_{k=0}^{n}\left(\begin{array}{c}
n \\
m
\end{array}\right)\left[A_{n-k}(\lambda)_{H} A_{k}^{(\alpha)}((1-\lambda) x, y ; \lambda)-\lambda A_{n-k}(\lambda)_{H} A_{k}^{(\alpha)}(x, y ; \lambda)\right] .
$$

Proof. Consider generating function (2.1), we have

$$
\begin{gathered}
\sum_{n=0}^{\infty} H_{H} A_{n}^{(\alpha)}(x, y ; \lambda) \frac{t^{n}}{n !}=\left(\frac{1-\lambda}{e^{t(\lambda-1)}-\lambda}\right)\left(\frac{e^{t(\lambda-1)}-\lambda}{1-\lambda}\right)\left(\frac{1-\lambda}{e^{t(\lambda-1)}-\lambda}\right)^{\alpha} e^{x t+y t^{2}} \\
=\frac{1}{1-\lambda}\left[\left(\frac{1-\lambda}{e^{t(\lambda-1)}-\lambda}\right) e^{(\lambda-1) t}\left(\frac{1-\lambda}{e^{t(\lambda-1)}-\lambda}\right)^{\alpha} e^{x t+y t^{2}}-\lambda\left(\frac{1-\lambda}{e^{t(\lambda-1)}-\lambda}\right)\left(\frac{1-\lambda}{e^{t(\lambda-1)}-\lambda}\right)^{\alpha} e^{x t+y t^{2}}\right] \\
=\frac{1}{1-\lambda}\left[\sum_{n=0}^{\infty} A_{n}(\lambda) \frac{t^{n}}{n !} \sum_{k=0}^{\infty} H_{k}^{(\alpha)}((\lambda-1) x, y ; \lambda) \frac{t^{k}}{k !}-\lambda \sum_{n=0}^{\infty} A_{n}(\lambda) \frac{t^{n}}{n !} \sum_{k=0}^{\infty} H_{k}^{(\alpha)}(x, y ; \lambda) \frac{t^{k}}{k !}\right] .
\end{gathered}
$$

Applying the Cauchy product rule in the above equation and then equating the coefficients of like powers of $t$ in both sides of the resultant equation, assertion (2.12) follows.

Theorem 2.8. For $0 \leq n \in \mathbb{N}$ and $p, q \in \mathbb{R}$, the following formula for ${ }_{H} A_{n}^{(\alpha)}(x, y ; \lambda)$ holds true:

$$
{ }_{H} A_{n}^{(\alpha)}(p x, q y ; \lambda)=n ! \sum_{k=0}^{n} \sum_{j=0}^{\left[\frac{k}{2}\right]} H_{n-k}^{(\alpha)}(x, y ; \lambda)((p-1) x)^{k-2 j}((q-1) y)^{j} \frac{t^{n}}{(n-k-2 j) ! j ! k !} .
$$

Proof. Rewrite the generating function (2.1), we have

$$
\begin{aligned}
I & =\sum_{n=0}^{\infty} H_{n}^{(\alpha)}(p x, q y ; \lambda) \frac{t^{n}}{n !}=\left(\frac{1-\lambda}{e^{(\lambda-1) t}-\lambda}\right)^{\alpha} e^{x t+y t^{2}} e^{(p-1) x t} e^{(q-1) y t^{2}} \\
& =\left(\sum_{n=0}^{\infty} H A_{n}^{(\alpha)}(x, y ; \lambda) \frac{t^{n}}{n !}\right)\left(\sum_{k=0}^{\infty}((p-1) x)^{k} \frac{t^{k}}{k !}\right)\left(\sum_{j=0}^{\infty}((q-1) y)^{j} \frac{t^{2 j}}{j !}\right) \\
& =\left(\sum_{n=0}^{\infty} H_{n}^{(\alpha)}(x, y ; \lambda) \frac{t^{n}}{n !}\right)\left(\sum_{k=0}^{\infty} \sum_{j=0}^{\infty}((p-1) x)^{k}((q-1) y)^{j} \frac{t^{k+2 j}}{n ! k ! j !}\right)
\end{aligned}
$$

replacing $k$ by $k-2 j$ in above equation, then

$$
\begin{aligned}
I & =\left(\sum_{n=0}^{\infty} H A_{n}^{(\alpha)}(x, y ; \lambda) \frac{t^{n}}{n !}\right)\left(\sum_{k=2 j}^{\infty}((p-1) x)^{k-2 j}((q-1) y)^{j} \frac{t^{k}}{(k-2 j) ! j !}\right) \\
& =\sum_{n=0}^{\infty} \sum_{k=2 j}^{\infty} H_{n}^{(\alpha)}(x, y ; \lambda)((p-1) x)^{k-2 j}((q-1) y)^{j} \frac{t^{n+k}}{(k-2 j) ! j ! n !} .
\end{aligned}
$$


Again replacing $n$ by $n-k$ in the above equation, we have

$$
I=\sum_{n=0}^{\infty} \sum_{k=0}^{n} \sum_{j=0}^{\left[\frac{k}{2}\right]}{ }_{H} A_{n-k}^{(\alpha)}(x, y ; \lambda)((p-1) x)^{k-2 j}((q-1) y)^{j} \frac{t^{n}}{(n-k-2 j) ! j ! k !} .
$$

Finally, equating the coefficients of $t^{n}$ on both sides, we acquire the result (2.13).

Theorem 2.9. For $n \in \mathbb{N}, p, q \in \mathbb{R}$ and $x, y \in \mathbb{C}$, then we have

$$
{ }_{H} A_{n}^{(\alpha)}(p x, q y ; \lambda)=\sum_{k=0}^{n}\left(\begin{array}{c}
n \\
m
\end{array}\right){ }_{H} A_{n-k}^{(\alpha)}(x, y ; \lambda) H_{k}((p-1) x,(q-1) y) .
$$

\section{Summation formulae for Hermite-based Apostol type Frobenius-Euler polynomials}

In this section, we provide implicit formulae, the Stirling numbers of the second kind and some relationships for Hermite-based Frobenius type Eulerian polynomials of order $\alpha$ associated with Apostol type Bernoulli, Genocchi and Euler polynomials. We now begin with the theorem given below.

Theorem 3.1. The following summation equality for Hermite-based Frobenius type Eulerian polynomials ${ }_{H} A_{n}^{(\alpha)}(x, y ; \lambda)$ of order $\alpha$ holds true:

$$
{ }_{H} A_{k+l}^{(\alpha)}(z, y ; \lambda)=\sum_{n, m=0}^{k, l}\left(\begin{array}{c}
l \\
m
\end{array}\right)\left(\begin{array}{l}
k \\
n
\end{array}\right)(z-x)^{n+m}{ }_{H} A_{k+l-n-m}^{(\alpha)}(x, y ; \lambda) .
$$

Proof. We replace $t$ by $t+w$ and rewrite the generating function (2.1) as

$$
\left(\frac{1-\lambda}{e^{(\lambda-1)(t+w)}-\lambda}\right)^{\alpha} e^{y(t+w)^{2}}=e^{-x(t+w)} \sum_{k, l=0}^{\infty} H_{H} A_{k+l}^{(\alpha)}(x, y ; \lambda) \frac{t^{k}}{k !} \frac{w^{l}}{l !}, \quad(\text { see }[6,14,15]) .
$$

Changing $x$ by $z$ in the equality above and then equating the resulting equation, we attain

$$
e^{(z-x)(t+w)} \sum_{k, l=0}^{\infty}{ }_{H} A_{k+l}^{(\alpha)}(x, y ; \lambda) \frac{t^{k}}{k !} \frac{w^{l}}{l !}=\sum_{k, l=0}^{\infty}{ }_{H} A_{k+l}^{(\alpha)}(z, y ; \lambda) \frac{t^{k}}{k !} \frac{w^{l}}{l !} .
$$

On expanding exponential function (3.3) yields

$$
\sum_{N=0}^{\infty} \frac{[(z-x)(t+w)]^{N}}{N !} \sum_{k, l=0}^{\infty}{ }_{H} A_{k+l}^{(\alpha)}(x, y ; \lambda) \frac{t^{k}}{k !} \frac{w^{l}}{l !}=\sum_{k, l=0}^{\infty}{ }_{H} A_{k+l}^{(\alpha)}(z, y ; \lambda) \frac{t^{k}}{k !} \frac{w^{l}}{l !},
$$

which on applying formula $[17, p .52(2)]$

$$
\sum_{N=0}^{\infty} f(N) \frac{(x+y)^{N}}{N !}=\sum_{n, m=0}^{\infty} f(n+m) \frac{x^{n}}{n !} \frac{y^{m}}{m !}
$$

in the left hand side becomes

$$
\sum_{n, m=0}^{\infty} \frac{(z-x)^{n+m} t^{n} w^{m}}{n ! m !} \sum_{k, l=0}^{\infty}{ }_{H} A_{k+l}^{(\alpha)}(x, y ; \lambda) \frac{t^{k}}{k !} \frac{w^{l}}{l !}=\sum_{k, l=0}^{\infty}{ }_{H} A_{k+l}^{(\alpha)}(z, y ; \lambda) \frac{t^{k}}{k !} \frac{w^{l}}{l !} .
$$

Here replacing $k$ by $k-n$, and $l$ by $l-m$ in the left hand side of (3.6), we obtain

$$
\sum_{k, l=0}^{\infty} \sum_{n, m=0}^{k, l} \frac{(z-x)^{n+m}}{n ! m !} H_{H}^{(\alpha)} A_{k+l-n-m}^{(x)}(x, y ; \lambda) \frac{t^{k}}{(k-n) !} \frac{w^{l}}{(l-m) !}=\sum_{k, l=0}^{\infty}{ }_{H} A_{k+l}^{(\alpha)}(z, y ; \lambda) \frac{t^{k}}{k !} \frac{w^{l}}{l !} .
$$


Lastly on comparing the coefficients of the like powers of $t$ and $w$ in the equation above, we derive the desired result.

Corollary 3.1. The following summation formula for Hermite-based Frobenius type Eulerian polynomials ${ }_{H} A_{n}^{(\alpha)}(x, y ; \lambda)$ of order $\alpha$ holds true:

$$
{ }_{H} A_{k}^{(\alpha)}(z, y ; \lambda)=\sum_{n=0}^{k}\left(\begin{array}{l}
k \\
n
\end{array}\right)(z-x)^{n}{ }_{H} A_{k-n}^{(\alpha)}(x, y ; \lambda) .
$$

Remark 3.1. Substituting $z$ by $z+x$ and choosing $y=0$ in Theorem (3.1), we obtain the following formula involving Hermite-based Frobenius type Eulerian polynomials ${ }_{H} A_{n}^{(\alpha)}(x, y ; \lambda)$ of one variable

$$
{ }_{H} A_{k+l}^{(\alpha)}(z+x ; \lambda)=\sum_{n, m=0}^{k, l}\left(\begin{array}{c}
l \\
m
\end{array}\right)\left(\begin{array}{l}
k \\
n
\end{array}\right) z^{n+m}{ }_{H} A_{k+l-n-m}^{(\alpha)}(x ; \lambda),
$$

while by taking $z=0$ in Theorem 3.1, we then acquire another formula involving Hermite-based Frobenius type Eulerian polynomials ${ }_{H} A_{n}^{(\alpha)}(x, y ; \lambda)$ of one and two variables

$$
{ }_{H} A_{k+l}^{(\alpha)}(y ; \lambda)=\sum_{n, m=0}^{k, l}\left(\begin{array}{l}
k \\
n
\end{array}\right)\left(\begin{array}{c}
l \\
m
\end{array}\right) H_{k+l-n-m}^{(\alpha)}(x, y ; \lambda)(-x)^{n+m}
$$

Theorem 3.2. The following summation identity for Hermite-based Frobenius type Eulerian polynomials ${ }_{H} A_{n}^{(\alpha)}(x, y ; \lambda)$ of order $\alpha$ holds true:

$$
{ }_{H} A_{n}^{(\alpha+1)}(x, y ; \lambda)=\sum_{m=0}^{n}\left(\begin{array}{c}
n \\
m
\end{array}\right) A_{n-m}(\lambda)_{H} A_{m}^{(\alpha)}(x, y ; \lambda) .
$$

Proof. From (2.1), we have

$$
\begin{aligned}
\frac{1-\lambda}{e^{(\lambda-1) t}-\lambda}\left(\frac{1-\lambda}{e^{(\lambda-1) t}-\lambda}\right)^{\alpha} e^{x t+y t^{2}} & =\frac{1-\lambda}{e^{(\lambda-1) t}-\lambda} \sum_{m=0}^{\infty} H_{m}^{(\alpha)}(x, y ; \lambda) \frac{t^{m}}{m !} \\
\left(\frac{1-\lambda}{e^{(\lambda-1) t}-\lambda}\right)^{\alpha+1} e^{x t+y t^{2}} & =\frac{1-\lambda}{e^{(\lambda-1) t}-\lambda} \sum_{m=0}^{\infty} H_{m}^{(\alpha)}(x, y ; \lambda) \frac{t^{m}}{m !} \\
& =\sum_{n=0}^{\infty} A_{n}(\lambda) \frac{t^{n}}{n !} \sum_{m=0}^{\infty} H_{m}^{(\alpha)}(x, y ; \lambda) \frac{t^{m}}{m !} .
\end{aligned}
$$

Now changing $n$ by $n-m$ and comparing the coefficients of $t^{n}$ leads to formula (3.11).

Theorem 3.3. The following summation formulae for Hermite-based Frobenius type Eulerian polynomials ${ }_{H} A_{n}^{(\alpha)}(x, y ; \lambda)$ of order $\alpha$ holds true:

$$
{ }_{H} A_{n}^{(\alpha)}(x+1, y ; \lambda)=\sum_{k=0}^{n}\left(\begin{array}{l}
n \\
k
\end{array}\right){ }_{H} A_{k}^{(\alpha)}(x, y ; \lambda) .
$$

Proof. Using definition (2.1), we have

$$
\begin{gathered}
\sum_{n=0}^{\infty} H A_{n}^{(\alpha)}(x+1, y ; \lambda) \frac{t^{n}}{n !}=\left(\frac{1-\lambda}{e^{(\lambda-1) t}-\lambda}\right)^{\alpha} e^{x t+y t^{2}} e^{t} \\
=\left(\sum_{k=0}^{\infty} H A_{k}^{(\alpha)}(x, y ; \lambda) \frac{t^{k}}{k !}\right)\left(\sum_{n=0}^{\infty} \frac{t^{n}}{n !}\right)=\sum_{n=0}^{\infty} \sum_{k=0}^{n}\left(\begin{array}{l}
n \\
k
\end{array}\right){ }_{H} A_{k}^{(\alpha)}(x, y ; \lambda) \frac{t^{n}}{n !} .
\end{gathered}
$$

Lastly, comparing the coefficients of the like powers of $t^{n}$, we get (3.12). 
Theorem 3.4. The following relation

$$
{ }_{H} A_{n}^{(\alpha)}(x, y ; \lambda)=\sum_{j=0}^{n}\left(\begin{array}{c}
n \\
j
\end{array}\right) \sum_{k=0}^{j}\left(\begin{array}{c}
-\alpha \\
k
\end{array}\right) k !(-1)^{k} S_{2}(j, k)(\lambda-1)^{j-k} H_{n-j}(x, y)
$$

holds true.

Proof. In view of (2.1), we get

$$
\begin{aligned}
& \sum_{n=0}^{\infty} H_{n}^{(\alpha)}(x, y ; \lambda) \frac{t^{n}}{n !}=\left(\frac{1-\lambda}{e^{t(\lambda-1)}-\lambda}\right)^{\alpha} e^{x t+y t^{2}}=e^{x t+y t^{2}}\left(1+\frac{e^{t(\lambda-1)}-1}{1-\lambda}\right)^{-\alpha} \\
& =\sum_{k=0}^{\infty}\left(\begin{array}{c}
-\alpha \\
k
\end{array}\right)\left(\frac{e^{t(\lambda-1)}-1}{1-\lambda}\right)^{k}\left(\sum_{n=0}^{\infty} H_{n}(x, y) \frac{t^{n}}{n !}\right) \\
& =\sum_{k=0}^{\infty}\left(\begin{array}{c}
-\alpha \\
k
\end{array}\right) \frac{k !}{(1-\lambda)^{k}} \sum_{n=0}^{\infty} S_{2}(n, k)(\lambda-1)^{n} \frac{t^{n}}{n !}\left(\sum_{n=0}^{\infty} H_{n}(x, y) \frac{t^{n}}{n !}\right) \\
& =\sum_{n=0}^{\infty}\left(\sum_{k=0}^{n}\left(\begin{array}{c}
-\alpha \\
k
\end{array}\right) k !(-1)^{k} S_{2}(n, k)(\lambda-1)^{n-k}\right) \frac{t^{n}}{n !}\left(\sum_{n=0}^{\infty} H_{n}(x, y) \frac{t^{n}}{n !}\right) \\
& =\sum_{n=0}^{\infty}\left(\sum_{j=0}^{n}\left(\begin{array}{c}
n \\
j
\end{array}\right) \sum_{k=0}^{j}\left(\begin{array}{c}
-\alpha \\
k
\end{array}\right) k !(-1)^{k} S_{2}(j, k)(\lambda-1)^{j-k} H_{n-j}(x, y)\right) \frac{t^{n}}{n !} .
\end{aligned}
$$

On comparing the coefficients of $\frac{t^{n}}{n !}$ in both sides, we get (3.13).

Theorem 3.5. The following formula:

$$
{ }_{H} A_{n}^{(\alpha)}(x, y ; \lambda)=\sum_{j=0}^{n} \sum_{k=0}^{n}\left(\begin{array}{c}
n \\
j
\end{array}\right)\left(\begin{array}{c}
\alpha+k-1 \\
k
\end{array}\right) k ! S_{2}(j, k)(\lambda-1)^{j-k} H_{n-j}(x, y)
$$

holds true.

Proof. By using generating function (2.1), we have

$$
\begin{aligned}
& \sum_{n=0}^{\infty}{ }_{H} A_{n}^{(\alpha)}(x, y ; \lambda) \frac{t^{n}}{n !}=\left(\frac{1-\lambda}{e^{t(\lambda-1)}-\lambda}\right)^{\alpha} e^{x t+y t^{2}}=\left(1+\frac{e^{t(\lambda-1)}-1}{1-\lambda}\right)^{-\alpha} e^{x t+y t^{2}} \\
& =\sum_{k=0}^{\infty}(-1)^{k}\left(\begin{array}{c}
\alpha+k-1 \\
k
\end{array}\right)\left(\frac{e^{t(\lambda-1)}-1}{1-\lambda}\right)^{k}\left(\sum_{n=0}^{\infty} H_{n}(x, y) \frac{t^{n}}{n !}\right) \\
& =\sum_{k=0}^{\infty}\left(\begin{array}{c}
\alpha+k-1 \\
k
\end{array}\right) \frac{k !}{(\lambda-1)^{k}} \sum_{n=0}^{\infty} S_{2}(n, k)(\lambda-1)^{n} \frac{t^{n}}{n !}\left(\sum_{n=0}^{\infty} H_{n}(x, y) \frac{t^{n}}{n !}\right) \\
& =\sum_{n=0}^{\infty}\left(\sum_{k=0}^{n}\left(\begin{array}{c}
\alpha+k-1 \\
k
\end{array}\right) k ! S_{2}(n, k)(\lambda-1)^{n-k}\right) \frac{t^{n}}{n !}\left(\sum_{n=0}^{\infty} H_{n}(x, y) \frac{t^{n}}{n !}\right) \\
& =\sum_{n=0}^{\infty}\left(\sum_{j=0}^{n} \sum_{k=0}^{n}\left(\begin{array}{c}
n \\
j
\end{array}\right)\left(\begin{array}{c}
\alpha+k-1 \\
k
\end{array}\right) k ! S_{2}(j, k)(\lambda-1)^{j-k} H_{n-j}(x, y)\right) \frac{t^{n}}{n !},
\end{aligned}
$$

which completes the proof.

Theorem 3.6. The following relation between the Hermite-based Frobenius type Eulerian polynomials ${ }_{H} A_{n}^{(\alpha)}(x, y ; \lambda)$ and Apostol type Bernoulli polynomials $B_{n}(x ; \lambda)$ holds true:

$$
{ }_{H} A_{n}^{(\alpha)}(x, y ; \lambda)=\sum_{k=0}^{n+1}\left(\begin{array}{c}
n+1 \\
k
\end{array}\right) \frac{{ }_{H} A_{n-k+1}^{(\alpha)}(0, y ; \lambda)}{n+1}\left(\lambda \sum_{r=0}^{k}\left(\begin{array}{c}
k \\
r
\end{array}\right) B_{k-r}(x ; \lambda)-B_{k}(x ; \lambda)\right) .
$$


Proof. Consider generating function (2.1), we have

$$
\begin{gathered}
\sum_{n=0}^{\infty}{ }_{H} A_{n}^{(\alpha)}(x, y ; \lambda) \frac{t^{n}}{n !}=\left(\frac{1-\lambda}{e^{t(\lambda-1)}-\lambda}\right)^{\alpha} e^{x t+y t^{2}}\left(\frac{t}{\lambda e^{t}-1}\right)\left(\frac{\lambda e^{t}-1}{t}\right) \\
=\frac{1}{t}\left(\lambda \sum_{n=0}^{\infty} H_{n}^{(\alpha)}(0, y ; \lambda) \frac{t^{n}}{n !} \sum_{k=0}^{\infty} B_{k}(x ; \lambda) \frac{t^{k}}{k !} \sum_{r=0}^{\infty} \frac{t^{r}}{r !}-\sum_{n=0}^{\infty}{ }_{H} A_{n}^{(\alpha)}(0, y ; \lambda) \frac{t^{n}}{n !} \sum_{k=0}^{\infty} B_{k}(x ; \lambda) \frac{t^{k}}{k !}\right) .
\end{gathered}
$$

On equating the coefficients of same powers of $t$ after using Cauchy product rule in (3.16), assertion (3.15) follows.

Theorem 3.7. The following relation between the Hermite-based Frobenius type Eulerian polynomials ${ }_{H} A_{n}^{(\alpha)}(x, y ; \lambda)$ and Apostol type Euler polynomials $E_{n}(x ; \lambda)$ holds true:

$$
{ }_{H} A_{n}^{(\alpha)}(x, y ; \lambda)=\sum_{k=0}^{n}\left(\begin{array}{l}
n \\
k
\end{array}\right) \frac{H A_{n-k}^{(\alpha)}(0, y ; \lambda)}{2}\left(\lambda \sum_{r=0}^{k}\left(\begin{array}{l}
k \\
r
\end{array}\right) E_{k-r}(x ; \lambda)+E_{k}(x ; \lambda)\right) .
$$

Proof. Consider generating function (2.1), we have

$$
\begin{gathered}
\sum_{n=0}^{\infty}{ }_{H} A_{n}^{(\alpha)}(x, y ; \lambda) \frac{t^{n}}{n !}=\left(\frac{1-\lambda}{e^{t(\lambda-1)}-\lambda}\right)^{\alpha} e^{x t+y t^{2}}\left(\frac{2}{\lambda e^{t}+1}\right)\left(\frac{\lambda e^{t}+1}{2}\right) \\
=\frac{1}{2}\left(\lambda \sum_{n=0}^{\infty} H_{n}^{(\alpha)}(0, y ; \lambda) \frac{t^{n}}{n !} \sum_{k=0}^{\infty} E_{k}(x ; \lambda) \frac{t^{k}}{k !} \sum_{r=0}^{\infty} \frac{t^{r}}{r !}+\sum_{n=0}^{\infty}{ }_{H} A_{n}^{(\alpha)}(0, y ; \lambda) \frac{t^{n}}{n !} \sum_{k=0}^{\infty} E_{k}(x ; \lambda) \frac{t^{k}}{k !}\right) .
\end{gathered}
$$

On comparing the coefficients of same powers of $t$ after using Cauchy product rule in (3.18), the desired result (3.17) follows.

Theorem 3.8. The following relation between the Hermite-based Frobenius type Eulerian polynomials ${ }_{H} A_{n}^{(\alpha)}(x, y ; \lambda)$ and Apostol type Genocchi polynomials $G_{n}(x ; \lambda)$ holds true:

$$
{ }_{H} A_{n}^{(\alpha)}(x, y ; \lambda)=\sum_{k=0}^{n+1}\left(\begin{array}{c}
n+1 \\
k
\end{array}\right) \frac{H A_{n-k+1}^{(\alpha)}(0, y ; \lambda)}{2(n+1)}\left(\lambda \sum_{r=0}^{k}\left(\begin{array}{l}
k \\
r
\end{array}\right) G_{k-r}(x ; \lambda)+G_{k}(x ; \lambda)\right) .
$$

Proof. Consider generating function (2.1), we have

$$
\begin{gathered}
\sum_{n=0}^{\infty} H_{n}^{(\alpha)}(x, y ; \lambda) \frac{t^{n}}{n !}=\left(\frac{1-\lambda}{e^{t(\lambda-1)}-\lambda}\right)^{\alpha} e^{x t+y t^{2}}\left(\frac{2 t}{\lambda e^{t}+1}\right)\left(\frac{\lambda e^{t}+1}{2 t}\right) \\
=\frac{1}{2 t}\left(\lambda \sum_{n=0}^{\infty} H_{n}^{(\alpha)}(0, y ; \lambda) \frac{t^{n}}{n !} \sum_{k=0}^{\infty} G_{k}(x ; \lambda) \frac{t^{k}}{k !} \sum_{r=0}^{\infty} \frac{t^{r}}{r !}+\sum_{n=0}^{\infty} H_{n}^{(\alpha)}(0, y ; \lambda) \frac{t^{n}}{n !} \sum_{k=0}^{\infty} G_{k}(x ; \lambda) \frac{t^{k}}{k !}\right) .
\end{gathered}
$$

On equating the coefficients of same powers of $t$ after using Cauchy product rule in above equation, we get (3.19).

\section{Identities for Hermite-based Frobenius type Eulerian polynomials}

In this part, we give general symmetry relations for the Hermite-based Frobenius type Eulerian polynomials ${ }_{H} A_{n}^{(\alpha)}(x, y ; \lambda)$ and extended Frobenius type Eulerian polynomials $A_{n}^{(\alpha)}(x ; \lambda)$ by using the generating functions (1.5) and (2.1). 
Theorem 4.1. Let $a, b,>0$ with $a \neq b$ and $x, y \in \mathbb{R}$. For $n \geq 0$, the following identity

$$
\begin{aligned}
& \sum_{k=0}^{n}\left(\begin{array}{l}
n \\
k
\end{array}\right) b^{k} a^{n-k}{ }_{H} A_{n-k}^{(\alpha)}\left(b x, b^{2} y ; \lambda\right)_{H} A_{k}^{(\alpha)}\left(a x, a^{2} y ; \lambda\right) \\
= & \sum_{k=0}^{n}\left(\begin{array}{l}
n \\
k
\end{array}\right) a^{k} b^{n-k}{ }_{H} A_{n-k}^{(\alpha)}\left(a x, a^{2} y ; \lambda\right)_{H} A_{k}^{(\alpha)}\left(b x, b^{2} y ; \lambda\right)
\end{aligned}
$$

holds true.

Proof. Let

$$
A(t)=\left(\frac{(1-\lambda)^{2}}{\left(e^{(\lambda-1) a t}-\lambda\right)\left(e^{(\lambda-1) b t}-\lambda\right)}\right)^{\alpha} e^{a b x t+a^{2} b^{2} y t^{2}} .
$$

The formula $A(t)$ is symmetric in $b$ and $a$, we then obtain

$$
\begin{aligned}
& A(t)=\sum_{n=0}^{\infty}{ }_{H} A_{n}^{(\alpha)}\left(b x, b^{2} y ; \lambda\right) \frac{(a t)^{n}}{n !} \sum_{k=0}^{\infty}{ }_{H} A_{k}^{(\alpha)}\left(a x, a^{2} y ; \lambda\right) \frac{(b t)^{k}}{k !} \\
= & \sum_{n=0}^{\infty}\left(\sum_{k=0}^{n}\left(\begin{array}{l}
n \\
k
\end{array}\right) b^{k} a^{n-k}{ }_{H} A_{n-k}^{(\alpha)}\left(b x, b^{2} y ; \lambda\right)_{H} A_{k}^{(\alpha)}\left(a x, a^{2} y ; \lambda\right)\right) \frac{t^{n}}{n !} .
\end{aligned}
$$

Analogously, we can derive

$$
\begin{aligned}
& A(t)=\sum_{n=0}^{\infty}{ }_{H} A_{n}^{(\alpha)}\left(a x, a^{2} x ; \lambda\right) \frac{(b t)^{n}}{n !} \sum_{k=0}^{\infty}{ }_{H} A_{k}^{(\alpha)}\left(b x, b^{2} y ; \lambda\right) \frac{(a t)^{k}}{k !} \\
= & \sum_{n=0}^{\infty}\left(\sum_{k=0}^{n}\left(\begin{array}{l}
n \\
k
\end{array}\right) a^{k} b^{n-k}{ }_{H} A_{n-k}^{(\alpha)}\left(a x, a^{2} y ; \lambda\right)_{H} E_{k}^{(\alpha)}\left(b x, b^{2} y ; \lambda\right)\right) \frac{t^{n}}{n !} .
\end{aligned}
$$

On comparing the coefficients of $t^{n}$ on the last two equations, we attain the desired result (4.1).

Remark 4.1. For $\alpha=1$ in Theorem 4.1,, the result reduces to

$$
\begin{aligned}
& \sum_{k=0}^{n}\left(\begin{array}{l}
n \\
k
\end{array}\right) b^{k} a^{n-k}{ }_{H} A_{n-k}\left(b x, b^{2} y ; \lambda\right)_{H} A_{k}\left(a x, a^{2} y ; \lambda\right) \\
= & \sum_{k=0}^{n}\left(\begin{array}{l}
n \\
k
\end{array}\right) a^{k} b^{n-k}{ }_{H} A_{n-k}\left(a x, a^{2} y ; \lambda\right)_{H} A_{k}\left(b x, b^{2} y ; \lambda\right) .
\end{aligned}
$$

Theorem 4.2. Let $a, b,>0$ with $a \neq b$ and $x, y \in \mathbb{R}$. For $n \geq 0$, the following identity

$$
\begin{aligned}
& \sum_{k=0}^{n}\left(\begin{array}{l}
n \\
k
\end{array}\right) \sum_{i=0}^{a-1} \sum_{j=0}^{b-1}(-\lambda)^{i+j} a^{n-k} b^{k}{ }_{H} A_{n-k}^{(\alpha)}\left(b x+\frac{b}{a} i+j, b^{2} z ; \lambda\right) A_{k}^{(\alpha)}(a y ; \lambda) \\
= & \sum_{k=0}^{n}\left(\begin{array}{l}
n \\
k
\end{array}\right) \sum_{i=0}^{b-1} \sum_{j=0}^{a-1}(-\lambda)^{i+j} b^{n-k} a_{H}^{k} A_{n-k}^{(\alpha)}\left(a x+\frac{a}{b} i+j, a^{2} z ; \lambda\right) A_{k}^{(\alpha)}(b y ; \lambda) .
\end{aligned}
$$

holds true. 
Proof. Consider the identity

$$
\begin{aligned}
B(t) & =\left(\frac{(1-\lambda)^{2}}{\left(e^{(\lambda-1) a t}-\lambda\right)\left(e^{(\lambda-1) b t}-\lambda\right)}\right)^{\alpha} \frac{1+\lambda(-1)^{a+1} e^{a b t}}{\left(\lambda e^{a t}+1\right)\left(\lambda e^{b t}+1\right)} e^{a b(x+y) t+a^{2} b^{2} z t^{2}} \\
& =\left(\frac{1-\lambda}{e^{(\lambda-1) a t}-\lambda}\right)^{\alpha} e^{a b x t+a^{2} b^{2} z t^{2}}\left(\frac{1-\lambda\left(-e^{-b t}\right)^{a}}{\lambda e^{b t}+1}\right)\left(\frac{1-\lambda}{e^{(\lambda-1) b t}-\lambda}\right)^{\alpha} e^{a b y t}\left(\frac{1-\lambda\left(-e^{-a t}\right)^{b}}{\lambda e^{a t}+1}\right) \\
& =\left(\frac{1-\lambda}{e^{(\lambda-1) a t}-\lambda}\right)^{\alpha} e^{a b x t+a^{2} b^{2} z t^{2}} \sum_{i=0}^{a-1}(-\lambda)^{i} e^{b t i}\left(\frac{1-\lambda}{e^{(\lambda-1) b t}-\lambda}\right)^{\alpha} e^{a b y t} \sum_{j=0}^{b-1}(-\lambda)^{j} e^{a t j} \\
& =\left(\frac{1-\lambda}{e^{(\lambda-1) a t}-\lambda}\right)^{\alpha} e^{a^{2} b^{2} z t^{2}} \sum_{i=0}^{a-1} \sum_{j=0}^{b-1}(-\lambda)^{i+j} e^{\left(b x+\frac{b}{a} i+j\right) a t} \sum_{k=0}^{\infty} A_{k}^{(\alpha)}(a y ; \lambda) \frac{(b t)^{k}}{k !}
\end{aligned}
$$

then

$$
\begin{aligned}
& B(t)=\sum_{n=0}^{\infty} \sum_{i=0}^{a-1} \sum_{j=0}^{b-1}(-\lambda)^{i+j}{ }_{H} A_{n}^{(\alpha)}\left(b x+\frac{b}{a} i+j, b^{2} z ; \lambda\right) \frac{(a t)^{n}}{n !} \sum_{k=0}^{\infty} A_{k}^{\alpha}(a y ; \lambda) \frac{(b t)^{k}}{(k) !} \\
& =\sum_{n=0}^{\infty} \sum_{k=0}^{n}\left(\begin{array}{l}
n \\
k
\end{array}\right) \sum_{i=0}^{a-1} \sum_{j=0}^{b-1}(-\lambda)^{i+j} a^{n-k} b^{k}{ }_{H} A_{n-k}^{(\alpha)}\left(b x+\frac{b}{a} i+j, b^{2} z ; \lambda\right) A_{k}^{(\alpha)}(a y ; \lambda) \frac{t^{n}}{n !} .
\end{aligned}
$$

On the other hand, we have

$$
B(t)=\sum_{n=0}^{\infty} \sum_{k=0}^{n}\left(\begin{array}{l}
n \\
k
\end{array}\right) \sum_{i=0}^{b-1} \sum_{j=0}^{a-1}(-\lambda)^{i+j} b^{n-k} a_{H}^{k} A_{n-k}^{(\alpha)}\left(a x+\frac{a}{b} i+j, a^{2} z ; \lambda\right) A_{k}^{(\alpha)}(b y ; \lambda) \frac{t^{n}}{n !} .
$$

Comparing the coefficients $t^{n}$ on the last two equations, we investigate the asserted result (4.4).

\section{REFERENCES}

[1] Bell, E. T, Exponential polynomials, Ann. of Math., 35(1934), 258-277.

[2] Carlitz, L, Eulerian numbers and polynomials, Math. Mag., 32(1959), 247-260.

[3] Carlitz, L, Eulerian numbers and polynomials of higher order, Duke Math. J., 27(1960), $401-423$.

[4] Choi, J, Kim, D. S, Kim, T and Kim, Y. H, A note on some identities of Frobenius-Euler numbers and polynomials, Inter. J. Math. Math. Sci., 2012(2012), 1-9.

[5] Dattoli, G, Lorenzutta, S and Cesarano, C, Finite sums and generalized forms of Bernoulli polynomials, Rendiconti di Mathematica, 19(1999), 385-391.

[6] Khan, W. A, Some properties of the generalized Apostol type Hermite-Based polynomials, Kyungpook Math. J., 55(2015), 597-614.

[7] Kim, D. S, Kim, T, Kim, Y. H, Dolgy, D. V, A note on Eulerian polynomials associated with Bernoulli and Euler numbers and polynomials, Adv. Stud. Contemp. Math., 22(2012), 379-389.

[8] Kim, D. S, Kim, T, Kim, W. J, Dolgy, D. V, A note on Eulerian polynomials, Abtr. Appl. Anal. (2012) Art. ID 269640, 10pp.

[9] Kim, D. S, Kim, T, Some new identities of Frobenius-Euler numbers and polynomials, J. Ineq. Appl., 2012(2012), 1-10.

[10] Kurt, B and Simsek, Y, On the generalized Apostol type Frobenius Euler polynomials, Advances in Differences equations, (2013), 1-9.

[11] Kurt, B and Simsek, Y, Frobenius Euler type polynomials related to Hermite-Bernoulli polynomials, Proceedings of the International Conferences on Numerical Analysis and Applied Mathematics, Amer, Inst. Phys. Conf. Proc., 1389(2011), 385-388. 
[12] Pathan, M. A and Khan, W. A, Some implicit summation formulas and symmetric identities for the generalized Hermite-based polynomials, Acta Universitatis Apulensis, 39(2014), 113-136.

[13] Pathan, M. A and Khan, W. A, Some implicit summation formulas and symmetric identities for the generalized Hermite-Bernoulli polynomials, Mediterr. J. Math., 12(2015), 679-695.

[14] Pathan, M. A and Khan, W. A, A new class of generalized polynomials associated with Hermite and Euler polynomials, Mediterr. J. Math., 13(2016), 913-928.

[15] Pathan, M.A and Khan, W. A, Some implicit summation formulas and symmetric identities for the generalized Hermite-Euler polynomials, East-West J. Maths., 16(1)(2014), 92-109.

[16] Srivastava, H. M, Eulerian and other integral representations for some families of hypergeometric polynomials, Inter. J. Appl. Math. Stat., 11(2007), 149-171.

[17] Srivastava, H. M and Manocha, H. L, A treatise on generating functions, Ellis Horwood Limited, New York, 1984.

[18] Srivastva, H. M, Boutiche, M. A, Rahmani, M, A class of Frobenius-type Eulerian polynomials, Rocky Mountain J. Math., 48(2018), 1003-1013.

[19] Simsek, Y, Generating functions for generalized Stirlings type numbers array type polynomials, Eulerian type polynomials and their application, Fixed Point Theory and Appl., doi:10.1186/16871812-2013-87, 2013 . 